\title{
A NEW ALGORITHM OF TRACKING TIME-VARYING CHANNELS IN IMPULSIVE NOISE ENVIRONMENT USING A ROBUST KALMAN FILTER
}

\author{
Z. G. Zhang, S. C. Chan and K. W. Tse \\ Department of Electrical and Electronic Engineering, The University of Hong Kong \\ Pokfulam Road, Hong Kong.
}

\begin{abstract}
This paper proposes a new algorithm for tracking timevarying channels in impulsive noise environment using a robust Kalman filter. It employs a simple dynamical model of the channel, where the changes in the impulse response coefficients are due entirely to the innovations of the Kalman filter. This reduces the arithmetic complexity, while offering reasonable good performance. The robust Kalman filter is used to restrain the adverse effect of impulsive noise and provide estimates of the covariance matrices of the state and measurement noises. The noisy channel estimates from the Kalman filter can be used to estimate the parameters of the channel coefficients when they are assumed to follow an AR model. Finally, the two processes can be coupled together to further improve the performance. Simulation results show that the new algorithm gives more stable performance than the conventional methods under impulsive noise environment.
\end{abstract}

\section{INTRODUCTION}

Time-varying channels are frequently encountered in wireless communications and other applications and their estimation is an important subject of research. One commonly used approach is to model the impulse response coefficients of the time-varying channels as an autoregressive (AR) process. In other words, the dynamics of the system is described in terms of the AR model. Given the system outputs as the measurements and treating the state as the impulse response coefficients, the Kalman filter can be then employed to estimate the time varying channel coefficients. Since the AR parameters of the channel coefficients are in general unavailable, different methods for their joint estimation have been proposed. In [1], the AR parameters of the time-varying channels are estimated by the Kalman filter and a leastsquares (LS) or recursive least-squares (RLS) method is used to estimate the channel AR parameters. A coupled method combining the Kalman filter and LS estimation for the AR parameters was introduced in [2]. The concept was also proposed for the estimation of MIMO channels in [3, 4]. Because Kalman filter and LS estimation algorithm are both sensitive to impulsive noise, their performance will be degraded in this non-Gaussian environment. To solve the problem, Fung and Chan [5] used a recursive least M-estimation (RLM) algorithm, instead of the RLS algorithm, to suppress the adverse effect of outliers.

In the algorithms of [1], instantaneous approximation is used as the expected value in the estimation of the $A R$ parameters in order to make it mathematically tractable. An important disadvantage of this approximation is that considerably number of samples is usually required to obtain reliable estimates of these quantities. This poses significant problem in time-varying environment as the quantities are changing rather rapidly with time. Since the estimated AR parameters, using the RLS or RLM algorithm, will be used as the state transition matrix of the Kalman filter, the channel coefficients estimated by the Kalman filter will be degraded considerably, especially in fast time-varying environment. Although the extended Kalman filter (EKF) can be employed to estimate the channel coefficients and the state-space method together, their convergence and performance are usually limited, because of the problem of estimating a number of model parameters from limited number of observations. Moreover, the EKF algorithm also requires high arithmetic complexity. It should be noted that more recent generalizations of the Kalman filter, such as the particle filters [8], can give good results, they are limited to low dimensions and require very high arithmetic complexity, which is undesirable for real-time applications.

In this paper, we propose a new algorithm for estimating time-varying channels under impulsive noise environment using a new robust Kalman filter. Instead of assuming that the impulse responses follow a complicated AR model, we only assume that the channel impulse responses are closely related to the previous one and model the changes as the innovation to the dynamical equation in the Kalman filter. In other words, the state transition matrix is an identity matrix and the changes of the channels are all caused by the innovation or state noise having an appropriate, usually slightly larger, variance. A 
robust Kalman filter, based on M-estimation, is employed to combat the adverse effect of the impulsive outliers. Then, the channel coefficients estimated by the robust Kalman filter are used to estimate the parameters of a low order AR model. Finally, these two estimations can be further coupled together as in [2] at each time instant to estimate the channel and AR parameters iteratively. The uncoupled version of the proposed algorithm has a low implementation complexity and reasonably good performance. The use of the robust Kalman filter improves the robustness of the algorithm in impulsive noise environment. It also provides estimates of the covariance matrices of the state and measure noises.

The paper is organized as follows. Section 2 describes the proposed channel model. Section 3 is devoted to time-varying channel estimation using our new Kalman filter. Section 4 discusses the estimation of AR parameters. Simulation results are presented in Section 5 and conclusions are drawn in Section 6.

\section{CHANNEL MODEL AND ESTIMATION}

Consider the identification of an unknown system with a time-varying impulse response $w_{k}(n), k=1,2, \ldots, L$. The system input $x(n)$, which is known as the training symbols in communications, is applied to the unknown system and the output $y(n)$ is observed to identify $w_{k}(n)$. Mathematically, we have at the $n$-th time instant:

$$
y(n)=\sum_{k=1}^{L} w_{k}(n) x(n-k+1)+\varepsilon(n),
$$

or in matrix form:

$$
y(n)=\mathbf{W}^{T}(n) \mathbf{X}(n)+\varepsilon(n),
$$

where $\varepsilon(n)$ is the additive white Gaussian noise with zero mean, $\mathbf{W}(n)=\left[w_{1}(n), \ldots, w_{L}(n)\right]^{T}$ is the time-varying channel vector and $\mathbf{X}(n)=[x(n), \ldots, x(n-L+1)]^{T}$ is the input data vector. For time-invariant channel $w_{k}(n)=w_{k}$, which is independent of time index $n$, it can be estimated by solving a LS problem to combat the effect of the additive noise using consecutive measurements $y(n)$. If the channel is time-varying, the problem is more difficult because the way how $w_{k}(n)$ change with time is generally unknown and the number of observations that $w_{k}(n)$ remain stationary might be limited. Conceptually, a better method for determining $w_{k}(n)$ would require some model or prior information. For example, in most reported works, $w_{k}(n)$ is modeled as an AR process. For instance, if a first order AR model is used, we have

$$
w_{k}(n+1)=\rho_{k} w_{k}(n)+\eta_{k}(n),
$$

and the state dynamic can be written as:

$$
\mathbf{W}(n+1)=\mathbf{F} \cdot \mathbf{W}(n)+\boldsymbol{\eta}(n)
$$

where $\mathbf{F}=\operatorname{diag}\left(\rho_{1}, \ldots, \rho_{L}\right)$ and $\boldsymbol{\eta}(n)=\left[\eta_{1}, \ldots, \eta_{L}\right]^{T}$. As mentioned earlier, the estimation of the AR parameters in form of $\mathbf{F}(n)$ increases the arithmetic complexity and rely on a more stringent assumption that $w_{k}(n)$ follow the AR model. In our proposed model, we assume that

$$
w_{k}(n+1)=w_{k}(n)+\eta_{k}(n),
$$

where $E\left[\boldsymbol{\eta}(n) \boldsymbol{\eta}^{T}(n)\right]=\mathbf{R}_{\eta}$. The physical meaning of this model is that $w_{k}(n)$ should be close to its previous value $w_{k}(n-1)$ and the variations of the channel is modeled as a Gaussian distributed state noise with covariance $\mathbf{R}_{\eta}$. The state transition matrix is thus an identity matrix. This simple model has the advantage of using fewer parameters. It is expected to yield lower arithmetic complexity and fast tracking speed but at the same time generating slightly higher estimation variance in $\mathbf{W}(n)$. To reduce this variance, $\mathbf{W}(n)$ will be estimated by another AR estimation process to be described in Section 4. The two processes can also be coupled together at each iteration to progressive refine the estimates.

Equation (2) and (3) constitute the following statespace equations:

$$
\begin{aligned}
& \mathbf{W}(n+1)=\mathbf{I} \cdot \mathbf{W}(n)+\boldsymbol{\eta}(n), \\
& y(n)=\mathbf{X}^{T}(n) \mathbf{W}(n)+\varepsilon(n) .
\end{aligned}
$$

This is in the same form as the Kalman filter formulation, where $\mathbf{W}(n)$ is the state to be estimated and (6a) is the state dynamic equation, while (6b) is the measurement equation. Thus, when $\varepsilon(n)$ and $\boldsymbol{\eta}(n)$ are Gaussian distributed with zero means and covariances $\sigma_{\varepsilon}$ and $\mathbf{R}_{\eta}$, then the optimal mean square error (MSE) estimator of $\mathbf{W}(n)$ is given by the Kalman filter.

Since the state equation of the proposed model is very simple, it also admits the following simple form:

$$
\left[\begin{array}{c}
\mathbf{W}(n-1) \\
y(n)
\end{array}\right]=\left[\begin{array}{c}
\mathbf{I} \\
\mathbf{X}^{T}(n)
\end{array}\right] \mathbf{W}(n)+\left[\begin{array}{c}
-\mathbf{\eta}(n) \\
\varepsilon(n)
\end{array}\right] .
$$

Let $\xi(n)=\left[-\boldsymbol{\eta}^{T}(n), \varepsilon(n)\right]^{T}$ and denote its covariance by $\mathbf{R}_{\xi}=\mathbf{S} \cdot \mathbf{S}^{T}$. The optimal LS estimate (which is also the Kalman filter solution) is

$$
\mathbf{W}(n)=\arg \min _{\mathbf{W}(n)}\left\|S^{-1}\left(\left[\begin{array}{c}
\mathbf{I} \\
\mathbf{X}^{T}(n)
\end{array}\right] \mathbf{W}(n)-\left[\begin{array}{c}
\mathbf{W}(n-1) \\
y(n)
\end{array}\right]\right)\right\|_{2} .
$$

The equations involve the identity matrix is equivalent to the diagonal loading in beam-forming and a regularized LS problem. The physical interpretation is that the state dynamic automatically imposes a smoothness constraint into the LS problem, which is very reasonable because we assume that the change is only due to the state noise $\boldsymbol{\eta}(n)$. Here, only one measurement equation is used, instead of using all the past measurement equations with 
exponential weighting. If the system is time-invariant, including more past measurements can help to reduce the variance and it will lead to the conventional RLS algorithm. For 'tracking' time-varying channels, this model with an appropriate selection of the covariance of the state noise is more attractive because of its good performance and low complexity. We now extend the proposed algorithm to the situation where $\varepsilon(n)$ contains impulsive outliers, which is frequently encountered in nature or from other man-made interference sources.

\section{CHANNEL ESTIMATION UNDER OUTLIERS}

We will use the robust Kalman filter method proposed in $[6,7]$ to solve the state-space equations in (6). $\mathrm{S}$ in (6) can be obtained by computing the Cholesky decomposition of $E\left[\xi(n) \xi^{T}(n)\right]$. Multiplying both sides of (7) by $\mathbf{S}^{-1}(n)$, we get

$$
\underline{\mathbf{Y}}(n)=\underline{\mathbf{X}}(n) \mathbf{W}(n)+\xi(n)
$$

where $\underline{\mathbf{X}}(n)=\mathbf{S}^{-1}(n)\left[\begin{array}{c}\mathbf{I} \\ \mathbf{X}^{T}(n)\end{array}\right], \quad \xi(n)=\left[\begin{array}{c}-\boldsymbol{\eta}(n) \\ \varepsilon(n)\end{array}\right], \quad$ and $\underline{\mathbf{Y}}(n)=\mathbf{S}^{-1}(n)\left[\begin{array}{c}\mathbf{W}(n-1) \\ y(n)\end{array}\right]$. It gives a linear LS problem as in (9) and the solution is given by:

$$
\mathbf{W}(n)=\left(\underline{\mathbf{X}}^{T}(n) \underline{\mathbf{X}}(n)\right)^{-1} \underline{\mathbf{X}}^{T}(n) \underline{\mathbf{Y}}(n),
$$

If the system is working under impulsive noise environment with $\varepsilon(n)$ corrupted by impulsive outliers, then the M-estimation should be employed instead of the LS criterion in (8)-(10) to combat their adverse effect. More precisely, the M-estimate of $\mathbf{W}(n)$ is given by:

$$
\mathbf{W}(n)=\arg \min _{\mathbf{W}(n)} \sum_{i=1}^{k} \rho\left(\underline{y}_{i}(n)-\underline{x}_{i}(n) \mathbf{W}(n)\right),
$$

where $\underline{y}_{i}(n)$ is the $i$-th element of $\underline{\mathbf{Y}}(n), \underline{x}_{i}{ }^{T}(n)$ is the $i$ th row of $\underline{\mathbf{X}}(k)$ and $\rho(\cdot)$ is a nonlinear score function which will restrain the adverse effect of outliers. We can use the iterative reweighted least-squares (IRLS) method to compute the solution of (11). The detailed algorithm can be found in [7] and is omitted here due to page limitation. It is worth-mentioning that the covariance of $\varepsilon(n)$ and $\boldsymbol{\eta}(n), \sigma_{\varepsilon}$ and $\sigma_{\eta}$ can be estimated as in [7].

Since our Kalman filter model is established under the assumption that $\mathbf{W}(n)$ follows the simple model in $(6 a)$, if $\mathbf{W}(n)$ follows a higher order $\mathrm{AR}$ model, then $\sigma_{\eta}$ should be chosen as a value larger than the innovation of the AR process in order to accommodate or model the actual change of the channel. Simulations show that an estimated value of $\sigma_{\eta}$ is 100 times larger than the actual covariance of $\boldsymbol{\eta}(n)$ and the estimation result is not too sensitive to this estimated values.

\section{AR PARAMETER ESTIMATION}

Suppose that the actual AR parameters of the channel $\mathbf{W}(n)$ is $\mathbf{\Lambda}(p)$, that is:

$$
\mathbf{W}(n)=\sum_{p=1}^{P} \mathbf{A}(p) \mathbf{W}(n-1)+\mathbf{u}(n),
$$

where $P$ is the order of the actual AR process, $\mathbf{u}(n)$ is an independent and identical distributed (i.i.d.) complex Gaussian noise with zero mean. From [1], we know the AR parameter can be estimated by the correlation matrices:

$$
\begin{aligned}
& R_{w}(\tau)=E\left\{\mathbf{W}(n) \mathbf{W}^{T}(n+\tau)\right\}, \\
& R_{w}^{H}(\tau)=\sum_{p=1}^{P} \mathbf{A}(p) R_{w}^{H}(\tau-p)+\sigma_{u}^{2} \delta(\tau) \mathbf{I}, \tau=1, \ldots, p .
\end{aligned}
$$

So, after we have estimated the channel, the correlation matrix $R_{w}(\tau)$ can be estimated using (13). The AR parameters can then be estimated from the noisy estimates of the robust Kalman filter by solving (14). The estimation can be further improved by combining the two processes together as in [2]. More precisely, once the AR parameters $\mathbf{A}(p ; n)$ at time instant $n$ are estimated, the estimated AR parameters and the model in (4) can be used to track the channel at time $n+1$. Simulation results show that it gives slightly better performance than using the uncoupled algorithms. The proposed algorithm (with coupling) is summarized as follows (without losing generality, we will assume the order of AR model is one): Initialize: At time $n=1$, assuming $\mathbf{A}(1)=\mathbf{I}$. The estimated covariance of $\boldsymbol{\eta}(n)$ is given a sufficiently large value.

Step 1: At time instant $n$, use the proposed robust Kalman filter and AR parameters $\mathbf{A}(n)$ to estimate the channel $\mathbf{W}(n+1)$.

Step 2: Estimate the AR parameters using LS. Calculate the correlation matrices: $R_{w}(0 ; n)=E\left\{\mathbf{W}(n) \mathbf{W}^{T}(n)\right\} \quad$ and $R_{w}(1 ; n)=E\left\{\mathbf{W}(n) \mathbf{W}^{T}(n+1)\right\}$.

Step 3: The updated AR parameter is: $\mathbf{A}(n+1)=R_{w}^{H}(1 ; n) / R_{w}^{H}(0 ; n)$. Go to $(1)$ with the updated $\mathbf{A}(n)$.

\section{SIMULATION RESULTS}

In our simulations, a first-order AR model with parameter $\mathbf{A}=\operatorname{diag}([0.95,0.98])$ is used to simulate the channel. The covariance of the measurement noise and the state noise are respectively $\sigma_{s}=0.01$ and $\sigma_{\eta}=0.01$. The SNR of the observation $y(n)$ is $20 \mathrm{~dB}$. Simulation results up to 200 time instants are displayed to illustrate the convergence performance of the various methods to the actual channel and the system parameters. For the sake of visualization, the impulsive noises with variance 100 are 
applied at time instants 100 and 150 . Simulation results of the method proposed in [1] is also included as a comparison. The channel estimation error and the AR parameter estimation error are both assessed by the normalized MSE: $\frac{1}{M C} \sum\|\mathbf{W}-\hat{\mathbf{W}}\|^{2} /\|\mathbf{W}\|^{2} \quad$ and $\frac{1}{M C} \sum\|\mathbf{A}-\hat{\mathbf{A}}\|^{2} /\|\mathbf{A}\|^{2}$, where $M C$ denotes the number of Monte-Carlo realizations. The learning curves of MSE are averaged over 100 Monte-Carlo realizations.

We can see from Fig. 1 and Fig. 2 that the proposed robust Kalman filter can combat the impulsive noise better than the proposed Kalman filter based on LS criterion obviously. We can still see that the algorithm coupled with channel estimation and AR parameter estimation only has a little better result than the uncoupled algorithm (without the AR parameter estimation). The AR parameter estimation is performed after time 32 so that enough measurements can be used to avoid divergence of the AR parameters estimation. Fig. 3 shows our proposed algorithm has a much better performance than the method in [1] in the estimation of AR parameters. It can also be seen from Fig. 3 that the robust Kalman filter can effectively restrain the adverse effect of impulsive noise.

\section{CONCLUSION}

A new algorithm for tracking time-varying channels in impulsive noise environment using a robust Kalman filter is presented. It employs a simple dynamical model of the channel to reduce the arithmetic complexity, while offering reasonable good performance. A robust Kalman filter is employed to restrain the adverse effect of impulsive noise. The noisy channel estimates from the Kalman filter can be used to estimate the parameters of the channel coefficients when they are governed by an AR model. Finally, the two processes can be coupled together to further improve the performance. Simulations show that the new algorithm gives more stable performance than the conventional methods under impulsive noise environment.

\section{REFERENCES}

[1] M. K. Tsatsanis, G. B. Giannakis and G. Zhou, "Estimation and equalization of fading channels with random coefficients", Signal Process., vol. 53, pp. 211-229, 1996.

[2] L.M. Davis, I.B. Collings and R.J. Evans, "Identification of time-varying linear channels"', in Proc. of the IEEE ICASSP, Munich, Germany, vol. 5, pp. 3921-3924, April 1997.

[3] C. Komninakis, C. Fragouli, A.H. Sayed and R.D. Wesel, "Adaptive multi-input multi-output fading channel equalization using Kalman estimation", in Proc. ICC 2000, vol. 3, pp. 16551659, New Orleans, Louisiana, June 18-22, 2000.
[4] M. Enescu, M. Sirbu, and V. Koivunen, "Recursive semiblind equalizer for time-varying MIMO channels", In IEEE Workshop on SSP, pp. 289-292, 2001.

[5] C. Y. Fung and S. C. Chan, "Estimation of fast fading channel in impulse noise environment", in Proc. of ISCAS 2002, vol. 4, pp. 497-500, 2002.

[6] Ž. M. Durović and B. D. Kovačević, "Robust estimation with unknown noise statistics," IEEE Transactions on Automatic Control, vol. 44, Issue 6, pp. 1292-1296, June 1999.

[7] S. C. Chan, Z. G. Zhang, and K. W. Tse, "A new robust Kalman filter algorithm under outliers and system uncertainties," in Proc. of IEEE ISCAS, Kobe, Japan, 2005.

[8] J. H. Kotecha and P. M. Djurić, "Gaussian sum particle filtering," IEEE Transactions on Signal Processing, vol. 51, no. 10 , pp. 2602-2612, Oct. 2003.

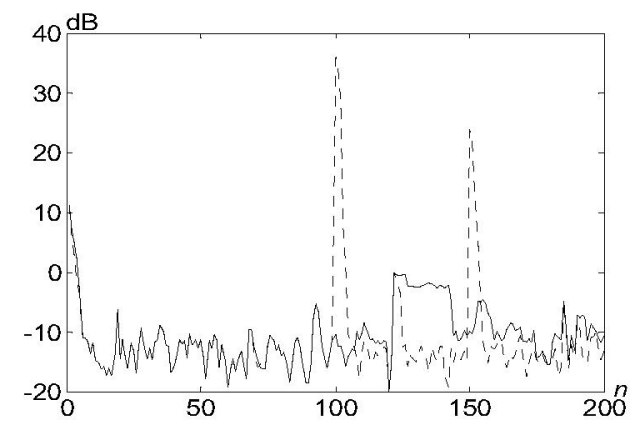

Fig. 1. MSE comparison of channel estimation (uncoupled algorithm). dotted line: proposed method based on LS criterion, solid line: proposed method based on M-estimation.

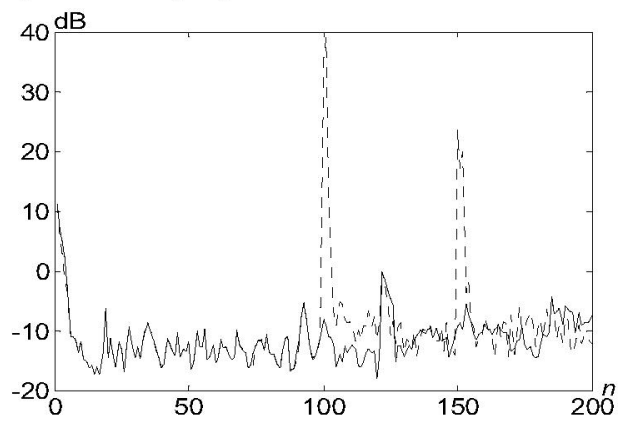

Fig. 1. MSE comparison of channel estimation (coupled algorithm). dotted line: proposed method based on LS criterion, solid line: proposed method based on M-estimation.



Fig. 2. MSE comparison of AR parameter estimation. dashed line: method proposed in [1], dotted line: proposed method based on LS criterion, solid line: proposed method based on M-estimation. 